African Crop Science Journal by African Crop Science Society is licensed under a Creative Commons Attribution 3.0 Uganda License. Based on a work at www.ajol.info/ and www.bioline.org.br/cs DOI: https://dx.doi.org/10.4314/acsj.v26i4.6

\title{
INFLUENCE DE L'APPORT DU BOIS RAMEALS FRAGMENTES ET DU FUMIER SUR L'INDICE DE LA QUALITE BIOLOGIQUE DU SOL, SUR UN SOL INCULTE, DANS UNE REGION SEMI-ARIDE MEDITERRANEENNE, ALGERIE
}

\author{
I. KERROUCHE, M. BENDJABALLAH ${ }^{1}$, K.E.D. BAZRI, M. GUTIRREZ LOPEZ ${ }^{2}$, \\ ZELTNI ABD ESSALEM ${ }^{3}$ et OUAHRANI GHANIA
}

Laboratoire d'écologie Dép.Eco .FSNV. Université Constantine1, Constantine, Algérie

${ }^{1}$ Laboratoire de Biosystématique et Ecologie des Arthropodes, Université de Constantine1, Constantine, Algérie

${ }^{2}$ Dép. de Zoologie et Anthropologie physique, Faculté de Biologie, Université de Complutense de Madrid,Ciudad Université, 28040 Madrid, Espagne

${ }^{3}$ Institut Technique des Grandes Cultures, Constantine, Algérie

Auteur correspondant: kerroucheibrahim@gmail.com

(Received 2 January, 2018; accepted 23 November, 2018)

\section{RÉSUMÉ}

La faune du sol est considérée comme un élément important des écosystèmes. Il peut être utilisé comme un élément d'évaluation de la qualité du sol et reflète l'état d'un sol. Il contribue à la régulation et à l'équilibre des caractéristiques physiques, chimiques et microbiologiques, et exprime mieux les variations et les changements écologiques pouvant affecter le sol. Le but de ce travail est de déterminé l'Indice de Qualité Biologique (QBS) d'un sol inculte amendé de BRF (Bois Raméal Fragmentés) et de fumier et en présence ou absence de vers de terre anécique (Octodrilus complanatus). La station d'étude est située en zone semi-aride (Constantine, Algérie). Ainsi, nous avons identifié 12 groupes de microarthropodes ou les acariens et les collemboles sont les plus abondants (82\%). La moyenne estimée du QBS est de $[16,7 \pm 0,8] ;[31,4 \pm 1,0] ;[32,3 \pm 0,8]$; $[32,9 \pm 1,4]$ et $[32,7 \pm 1,4]$ respectivement dans T (Témoin), BRF (Bois Raméal Fragmenté), BRFF (Bois Raméal Fragmenté et Fumier), BRFV (Bois Raméal Fragmenté et Vers de terre) et BRFFV (Bois Raméal Fragmenté, Fumier et Vers de terre), la différence est très significative entre les placettes étudiées. Par ailleurs, le QBS varie très significativement d'un mois à un autre. En outre, il existe un effet température très significatif sur le QBS. En effet plus la température s'élève et plus le QBS diminue. D'un point de vue pratique, nos résultats contribue à l'application du QBS et fournit des valeurs de référence pour cet indice pour des sols situés dans les zones semi-arides méditerranéennes.

Mots Clés: Indice de la qualité biologique du sol, bois raméal fragmentés, fumier, déchets organiques, microarthropodes et agroécologie

\begin{abstract}
Soil fauna is an important part of ecosystems. It can be used as an element to assess soil quality and reflects the state of soil, it also contributes to the regulation and balance of physical, chemical and microbiological characteristics, and expresses the variations and ecological changes that affect soil. The objective of this study was to determine the organic quality index (QBS) of an unreduced soil of Ramial Chipped Wood (RCW) and manure. This study was carried out on uncultivated soil of an experimental farm of the Technical Institute of Field Crops (ITGC) in
\end{abstract}


the region of El Khroub, Constantine, Algeria. The QBS method required the search for the most suitable soil morphological form (morpho-type) avoiding classification at the species level. Microarthropods were separated from the soil and weighed using the biological form approach to assess their level of adaptation to the soil environment. Twelve groups of microarthropods were identified, whereby mites and collembolans were the most representative (82\%). The estimated average QBS was [16.7 \pm 0.8$]$; [31.4 \pm 1.0$]$; [32.3 \pm 0.8$]$; [32.9 \pm 1.4$]$ and [32.7 \pm 1.4 ], respectively, in $\mathrm{T}$ (Control with no amendments), BRF (Rameal Chipped Wood), BRFF(Rameal Chipped Wood and Manure ), BRFV (Rameal Chipped Wood and Earth worms) and BRFFV (Rameal Chipped Wood, Manure and Earth worms). The difference was very significant between different types of plots. In addition, the QBS varied significantly from one month to another. The effect of temperature was significant on the quality of the QBS, the higher the temperature, the lower the QBS. From a practical point of view, the information reported contributes to the application of the QBS and provides reference values for this index in a soil of a semi-arid Mediterranean region, Constantine in Algeria.

Key Words: Agroecology, microarthropods, ramial chipped wood, soil biological index

\section{INTRODUCTION}

La faune du sol est considérée comme un élément important des écosystèmes (Davis, 1961; Edwards et Lofty, 1982; Hedlund et Öhrn, 2000; Wolters, 2000; Osler et Sommerkorn, 2007). En effet, elle est prise comme un bioindicateur indispensable pour évaluer la qualité des sols elle reflète l'état d'un sol (Stork et Eggleton, 1992; Buckerfield et al., 1997; Paoletti et Hassal, 1999 ; Yeates, 2003). En effet, la diversité de la pédofaune est un indice important pour la régulation et l'équilibre des caractéristiques physiques, chimiques et microbiologiques, et exprime mieux les variations et les changements écologiques qui peuvent affecter le sol (Eijsackers, 1983; Ekschmitt et al., 2001; Menta et al., 2008; Paolo et al., 2010). Ainsi, la faune du sol est le meilleur indicateur de la qualité du sol (Yan et al., 2012). Récemment, Parisi et al. (2005) ont introduit un indice simplifié basé sur les types des microarthropodes présents dans le sol. Cet indice ne nécessite pas une identification au niveau des espèces. "L'Indice de la Qualité Biologique du Sol » ou «Qualità Biologica del Suolo ». La méthode QBS nécessite la recherche de la forme biologique (morphotype) la plus adaptée au sol. Ce type recevra un score éco-morphologique (EMI) proportionnel à son niveau d'adaptation. En règle générale, les formes eu-édaphique (c'està-dire les formes qui vivent en profonde du
Sol-vivant) obtiennent une EMI égale à 20, les formes hémi-édaphiques (c'est-à-dire intermédiaires) reçoivent un indice proportionnel à leur degré de spécialisation, tandis que les formes épi-édaphique (qui vivent en surface) reçoivent un EMI = 1. Ainsi, pour calculer le QBS d'un échantillon, il suffit de faire la somme des EMI de cet échantillon (Parisi et al., 2005).

Par ailleurs, la présence de matière organique dans les sols agricoles est un facteur important pour évaluer l'état du sol. Le BRF et le Fumier sont des entrants énergétiques qui peuvent améliorer le sol. Le Bois Raméal Fragmenté (BRF) désigne l'ensemble de branches qui ont un diamètre inférieur à $7 \mathrm{~cm}$ (Lemieux, 1986). Ces branches se caractérisent par une teneur en composés phénoliques faible et une teneur en nutriments relativement élevée (Hendrickson, 1987; Edmonds, 1987). En outre, les BRF constituent une source importante de carbone pour les sols agricoles dégradés (Allison 1973). N'Dayegamiye et Angers (1993) ont observé une augmentation du carbone du sol à la suite de l'apport de BRF au sol. En générale, les amendements organiques riches en carbone, favorise la vie du sol (Tremblay et Beauchamp, 1998). Le fumier est une matière organique résultant des déjections (excréments et urine) d'animaux mélangées avec la litière, après compostage, on l'utilise comme fertilisant en agriculture, les fumiers contribuent au maintien de la fertilité des sols (Hiraoka et al., 2005). 
Les vers de terre lombriciens sont considérés comme très importants dans le recyclage de la matière organique du sol. Certaines espèces tels les vers de terre anécique comme Octodrilus complanatus, enterrent les résidus organiques superficiels, les dégradent et libèrent les éléments chimiques (N,P,K, etc.) qui y sont contenus(Gates, 1972). Les vers de terre anéciques sont de gros vers de terre, de couleur brune qui constituent le troisième type écologique (épigés, anéciques et endogés). Ce sont eux que l'on trouve lorsqu'on retourne la terre. S'il n'y a pas de vers de terre dans le sol ce n'est pas bon signe. Ainsi, la faune lombricienne est connue pour son importance capitale pour la "formation de la terre végétale". Le rôle mécanique des lombriciens est considérable ; il s'apparente à un labour, remarquable par ses qualités bien supérieures à celles des travaux aratoire classique (Bouche, 1974).

Notre travail, s'intéresse aux QBS d'un sol inculte auquel nous avons ajouté des entrants énergétiques du Bois Raméal Fragmentés (BRF) et/ou du Fumier et en présence/absence de vers de terre anécique de l'espèce Octodrilus complanatus. Nous avons introduit des lombriciens car ils permettent d'augmenter la vitesse de dégradation du BRF et du fumier. Ainsi, la présence de lombriciens, et d'entrants énergétiques (BRF et fumier) devraient influencer positivement le QBS du sol étudié. Enfin, nous avons déterminé le QBS car il est méconnu en zone semi- aride méditerranéenne en générale et en Algérie en particulier.

\section{MATÉRIELS ET MÉTHODES}

Ce travail est réalisé, sur l'une des terres incultes d'une ferme expérimentale de l'Institut Technique des Grandes Cultures (ITGC) dans la région d'El Khroub (Constantine, Algérie). La station d'étude est située en zone semiaride, dont la moyenne des précipitations annuelles est de $450 \mathrm{~mm} / \mathrm{an}$, une altitude de $594 \mathrm{~m}$, une latitude de 6.40 Est et une longitude de 36.16 Nord (Fig. 1).

Dans la station, il a été choisi une parcelle de $100 \mathrm{~m}^{2}$. Nous l'avons subdivisée en 15 placettes de $2 \mathrm{~m}^{2}$ chacune. La Figure 2, montre, 5 modalités des parcelles témoins $(\mathrm{T})$, des parcelles avec que du Bois Raméal fragmenté (BRF), des parcelles avec un mélange de Bois Raméal fragmenté et du

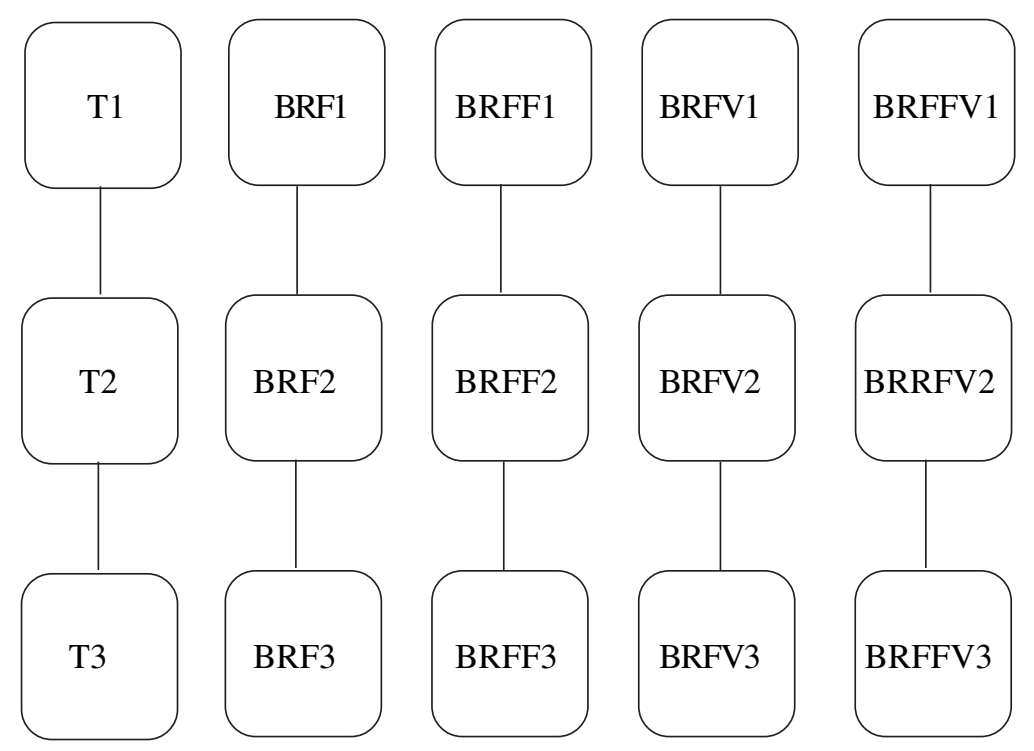

Figure 1. Plan expérimental. 
fumier (BRFF), des parcelles avec Bois Raméal fragmenté et vers de terre (BRFV) et des parcelles avec un mélange de BRF et de fumier et en présence de vers de terre (BRFFV). En outre pour chaque observation , 3 répétitions ont été faites.

Calcul des apports énergétiques (BRF et fumier) et présentation des placettes. Pour chaque placette étudiée à l'exception du témoin, un volume $0,03 \mathrm{~m}^{3} / \mathrm{m}^{2}$ a été apporté de BRF ou de fumier, ce qui équivaut à une quantité de $4 \mathrm{~kg} / \mathrm{m}^{2}$ soit une hauteur de $4 \mathrm{~cm}$ d'apport. En effet, le volume qu'il s'agit d'ajouter dans un hectare pour une épaisseur de $4 \mathrm{~cm}$ est de $40 \mathrm{t} \mathrm{ha}^{-1}$ (Caron et Lemieux, 1999). Il a été mis ainsi $8 \mathrm{~kg}$ d'entrant énergétique pour chaque parcelle. Le Tableau 1 donne la composition des différentes placettes étudiées dans notre expérimentation.

Choix du BRF. Nous avons choisi des petites branches inférieures à $7 \mathrm{~cm}$ de diamètre du nectarinier (Prunus persica var. nucipersica, C'est un Angiospermes et d'après Hendrickson, (1987) le contenu total en nutriments dans les rameaux est plus élevé chez les Angiospermes que chez les Gymnospermes.

Choix du fumier. Nous avons choisi comme fumier celui des bovins. Il a été incorporé au sol en automne après compostage.

Choix des vers de terre. Dans les parcelles BRFV et BRFFV, des vers de terre anéciques de l'espèce (Octodrilus complanatus) ont été introduits (Tableau 1). Leur introduction permet une meilleure décomposition des entrants énergétiques (Gates, 1972) et favorisent une plus grande porosité (Edwards et Bohlen, 1996).

\section{Paramètres étudiés}

Les paramètres physico-chimiques. Les paramètres physico-chimiques du sol déterminés sont le $\mathrm{pH}$, la conductivité

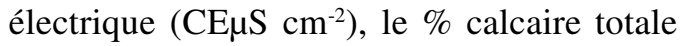
$\left(\% \mathrm{CaCO}_{3}\right)$, la porosité $(\% \mathrm{P})$ le $\%$ carbone total $(\% \mathrm{C})$, le taux D'azote totale $(\% \mathrm{~N})$. Les paramètres physico-chimiques ont été mesurés par des méthodes standards.

Les paramètres biotiques. Le Qualité Biologique du Sol indice (QBS) a été suivi mensuellement de mars 2016 jusqu'au mois de février 2017 sauf les mois de juin juillet et aout soit 9 mois de suivi. Les échantillons du sol ont été collectés à partir des différents types de parcelles étudiées (Fig. 2). Ces échantillons du sol ont été collectés à partir des différents types de placettes étudiées. Les prélèvements des échantillons ont été effectués dans les 10 premiers centimètres du sol et ils ont été mis dans des sachets de plastique pour les étudier au laboratoire.

\section{Détermination de l'indice de la qualité biologique du sol (QBS)}

Les principales étapes pour obtenir les valeurs QBS sont :

TABLEAU 1. Composition des placettes étudiées

\begin{tabular}{lll}
\hline Placettes & Composition & Symboles \\
\hline Témoin & Terrain nu & T1, T2 ; T3 \\
BRF & $8 \mathrm{~kg}$ de BRF. & BRF1, BRF2, BRF3 \\
BRFF & $6 \mathrm{~kg}$ de BRF, 2 kg de fumier, & BRFF1, BRFF2, BRFF3 \\
BRFV & $8 \mathrm{~kg}$ de BRF, et 120 vers de terre Octodrilus complanatus & BRFV1, BRFV2, BRFV3 \\
BRFFV & $\begin{array}{l}\text { 6 kg de BRF, 2 kg de fumier, et 120 vers de terre Octodrilus } \\
\text { complanatus }\end{array}$ & BRFFV1, BRFFV2, BRFFV3 \\
& & \\
\hline
\end{tabular}


(i) Échantillonnage. Dans le site d'étude, on délimite une zone représentative pour l'échantillonnage du sol. Pour le calcul du QBS les échantillons doivent être collectés lorsque l'humidité du sol se situe entre 40 et $80 \%$. Les échantillons sont placés dans des sacs en plastique étiquetés et fermés (Parisi et al., 2005).

(ii) Extraction des microarthropodes. Selon Bachelier (1978), les microarthropodes sont extraits par plusieurs méthodes, dont la plus simple et la plus utilisée à nos jours est celle connue sous le nom «extraction par voie sèche» décrite par Berlez-Tullgren (1918). La durée d'extraction (jamais inférieure à 5 jours) sera proportionnelle à la teneur en eau de l'échantillon de sol, telle que déterminée par (Parisi, 1974). Les échantillons extraits sont observés sous un stéréo-microscope à faible grossissement (45 fois, habituellement 20 à 40 fois suffisant) dans le même liquide conservateur (Parisi et al., 2005).

(iii) Détermination des formes biologiques et calcul de l'indice QBS. Définir les formes biologiques présentes dans un échantillon signifie reconnaître les différents niveaux d'adaptation à l'environnement du sol pour chaque groupe systématique. La méthode QBS nécessite la recherche de la forme biologique (morpho-type) la plus adaptée au sol. Ce type recevra un score écomorphologique (EMI) proportionnel à son niveau d'adaptation (Tableau 2). En règle générale, les formes eu-édaphique (c'està-dire les formes qui vivent en profonde du Sol-vivant) obtiennent une EMI $=20$, les formes hémi-édaphiques (c'est-à-dire intermédiaires) reçoivent un d'indice Proportionnel à leur degré de spécialisation, tandis que les formes épiédaphique (qui vivent en surface) reçoivent un $I E M=1$. Pour calculer le QBS d'un échantillon, il suffit de faire la somme des EMI de cet échantillon (Parisi et al., 2005) (Tableau 2).

TABLEAU 2. Indices Eco-Morphologiques (IEM) des groupes de microarthropodes édaphiques (Parisi $e t$ al., 2005)

\begin{tabular}{|c|c|}
\hline Groupe & EMI score \\
\hline Protura & 20 \\
\hline Diplura & 20 \\
\hline Collembola & $1-20$ \\
\hline Microcoryphie & 10 \\
\hline Zygentomata & 10 \\
\hline Dermaptera & 1 \\
\hline Orthoptères & $1-20$ \\
\hline Embioptera & 10 \\
\hline Blattaria & 5 \\
\hline Psocoptera & 1 \\
\hline Hemiptera & $1-10$ \\
\hline Thysanoptères & 1 \\
\hline Coleoptera & $1-20$ \\
\hline Hyménoptères & $1-5$ \\
\hline Diptères (larves) & 10 \\
\hline Autres insectes holométaboles (larves) & 10 \\
\hline Autres insectes holométaboles & 1 \\
\hline Acariens & 20 \\
\hline Aranéide & $1-5$ \\
\hline Opilion & 10 \\
\hline Palpigrade & 20 \\
\hline Pseudoscorpions & 20 \\
\hline Isopode & 10 \\
\hline Chilopode & $10-20$ \\
\hline Diplopode & $10-20$ \\
\hline Pauropode & 20 \\
\hline Symphyles & 20 \\
\hline
\end{tabular}

\section{RÉSULTATS}

(i) Nature du sol : Le Tableau.3, montre que la porosité est de $[34 \pm 1,51 \%]$; le $\mathrm{pH}$ de $[7,84 \pm 0,10]$, le taux de $\mathrm{CaCO} 3$ a une valeur de $[31,72 \pm 0,75 \%]$; pour la conductivité la moyenne est de $[34,1 \pm 3,12 \mu \mathrm{S} / \mathrm{cm}]$, le taux d'azote de $[0,18 \pm 0,00 \%]$, le taux de MO est de $[3,43 \pm 0,31 \%]$, quant au $\mathrm{C} / \mathrm{N}$ la moyenne est de $[12,71 \pm 0,88]$. 
(ii) Les groupes de microarthropodes identifiés. Nous avons identifié 12 groupes de microarthropodes (Tableau 4) dont les Acariens, Collemboles, Coléoptères, Hyménoptères, Araignée, Diptères, Hemiptera, Symphyles, Psocoptera, Blattaria, Chilopode et Diplopode . Ainsi, pendant la période d'étude, les acariens (49\%) et les Collemboles (34\%) sont les plus fréquemment présents, par contre les Coléoptères $(5,8 \%)$, les hyménoptères $(4,4 \%)$, les araignées $(3,5 \%)$ et larve de diptère $(1,5 \%)$ sont faiblement représentées et les Hemipteres, Symphyles, Psocopteres, Blattare, Chilopode et Diplopode sont franchement rare (Tableau 4).

(iii) Détermination de l'indice de la qualité biologique (QBS) des sols étudiés. Le Tableau 5, montre que la moyenne estimée du QBS est de $[16,7 \pm 0,8]$; $[31,4 \pm 1,0] ;[32,3 \pm 0,8] ;[32,9 \pm 1,4]$ et $[32,7 \pm 1,4]$ respectivement dans $\mathrm{T}, \mathrm{BRF}$, BRFF, BRFV et BRFFV. La différence est très significative (Fobs $=10,905 ; \mathrm{ddl}=4$ $\mathrm{P}<0,000$ ) (Tableau 6, Fig. 2). Le test de Tukey (Valeur critique du d de Tukey : 3,937 ) indique 2 modalités de QBS soit BRFV, BRFFV, BRFF et BRF (a) $>$ T (b).

TABLEAU 4. Fréquence (\%) des groupes des microarthropodes récoltés pendant la période d'étude

\begin{tabular}{lc}
\hline Microarthropodes & Fréquence (\%) \\
\hline Acari & 49,4 \\
Collembola & 34,4 \\
Coleoptera & 5,8 \\
Hyménoptèrea & 4,4 \\
Araneae & 3,5 \\
Diptera (larvae) & 1,5 \\
Hemiptera & 0,5 \\
Symphyla & 0,2 \\
Psocoptera & 0,2 \\
Blattaria & 0,1 \\
Chilopoda & 0,1 \\
Diplopoda & 0,1 \\
\hline
\end{tabular}


TABLEAU 5. Intervalle de confiance à $95 \%$ pour les moyennes estimées pour la variable QBS et leurs valeurs extrêmes entre parenthèses pour les parcelles étudiées

\begin{tabular}{lllll}
\hline $\mathrm{T}$ & BRF & BRFF & BRFV & BRFFV \\
\hline$[16,7 \pm 0,8]$ & {$[31,4 \pm 1,0]$} & {$[32,3 \pm 0,8]$} & {$[32,9 \pm 1,4]$} & {$[32,7 \pm 1,4]$} \\
$(0-40)$ & $(0-61)$ & $(0-50)$ & $(0-75)$ & $(0-71)$ \\
\hline
\end{tabular}

TABLEAU 6. Anova pour la variable QBS

\begin{tabular}{lrccrr}
\hline Source & DDL & Somme des carrés & Moyenne des carrés & $F$ & Pr $>$ F \\
\hline Parcelles & 4 & 5295,407 & 1323,852 & 10,905 & $<0,0001$ \\
Mois & 8 & 15124,133 & 1890,517 & 15,573 & $<0,0001$ \\
Parcelles*mois & 32 & 7171,793 & 224,119 & 1,846 & 0,013 \\
\hline
\end{tabular}

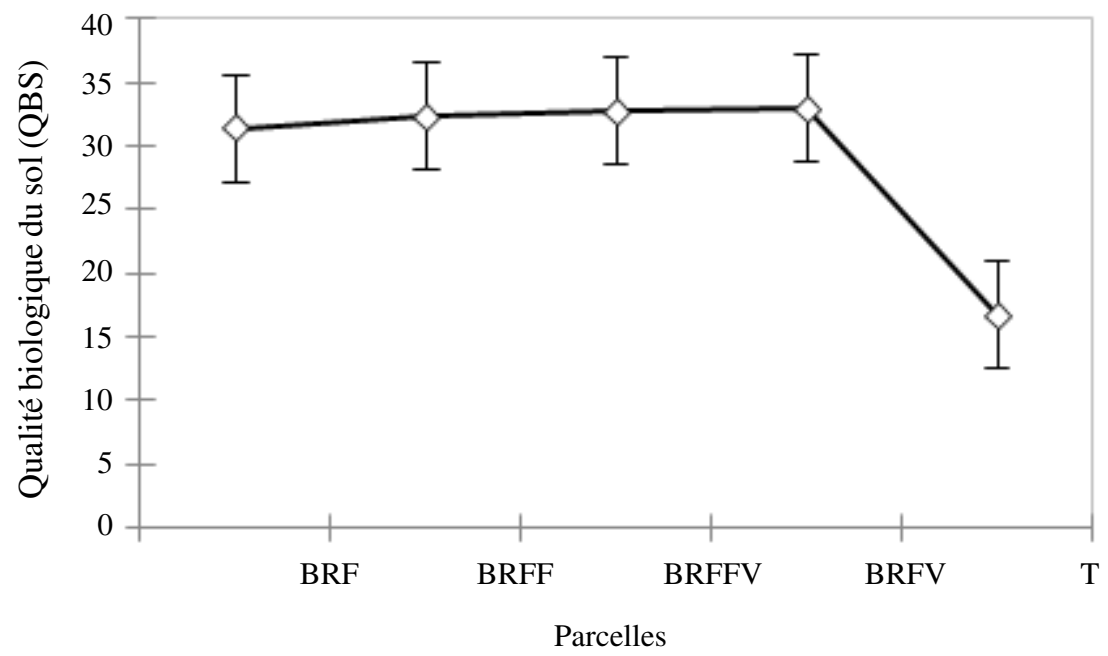

Figure 2. Variation moyenne du QBS dans les parcelles étudiées.

(iv) Détermination de QBS selon les mois. Les QBS calculé pour les mois étudiés la moyenne estimée est de $[23,4 \pm 1,6]$; $[7 \pm 1,6] ; \quad[23,2 \pm 0,8] ; \quad[33,0 \pm 1,7]$; $[44,4 \pm 2,4] ;[24,1 \pm 1,6] ;[31,3 \pm 1,7]$; $[39,8 \pm 2,5]$ et $[36,5 \pm 1,1]$ respectivement pour le mois de Mars; Avril; Mai; Septembre, Octobre, Novembre, Décembre, Janvier et Février (Tableau 7). La différence est très significative (Fobs= 15,573; ddl= $8 \mathrm{P}<0,000$ ) (Tableau 6, Fig. 3 ). Le test de Tukey (Valeur critique du d de Tukey :4,495 ) indique 8 modalités de QBS soit : Octobre (a)>Janvier (ab )> Février $(a b c)>$ Septembre $($ abcd $)>$ Décembre (bcd) $>$ Novembre (cd) $>$ Mars, Mai ( d) $>$ Avril (e). Par ailleurs, il y a un effet Parcelles*Mois (Fobs $=1,846$; $\mathrm{ddl}=32 \mathrm{P}<0,013$ ) (Tableau 7, Fig. 4) cela signifie que le QBS est influencé par la qualité de la parcelle mais aussi par les facteurs climatique tels que les précipitations et les températures. 


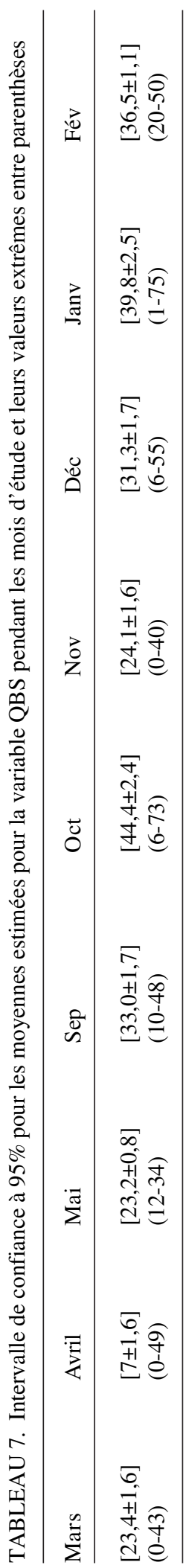

I. KERROUCHE et al.

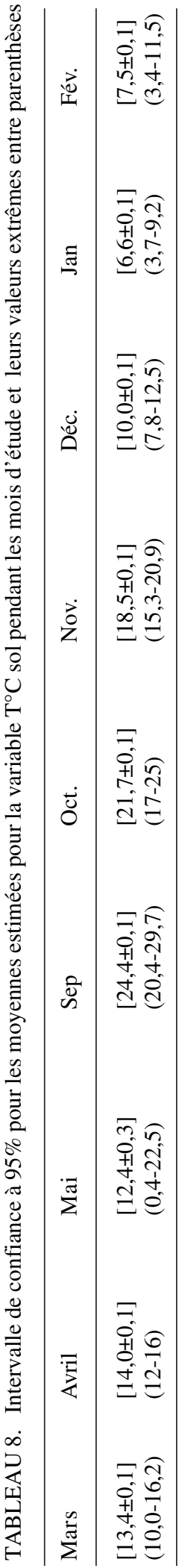

(v) Effet de la $\mathbf{T}^{\circ} \mathbf{C}$ sol sur le QBS. Le Tableau 8 , montre que les moyennes estimées de la $\mathrm{T}^{\circ} \mathrm{C}$ sol sont de $[13,4 \pm 0,1]$; $[14,0 \pm 0,1] ;[12,4 \pm 0,3] ;[24,4 \pm 0,1]$; $[21,7 \pm 0,1] ;[18,5 \pm 0,1] ;[10,0 \pm 0,1]$; $[6,6 \pm 0,1]$ et $[7,5 \pm 0,1]^{\circ} \mathrm{C}$ respectivement pour Mars, Avril, Mai, Septembre, Octobre, Novembre, Décembre, Janvier et Février. La différence entre les moyennes des températures du sol est très significative (Fobs $=151,144 ; \mathrm{ddl}=8$ et $\mathrm{P}<0,000$ ) (Tableau 9). Cela signifie que les températures varient mensuellement. Par ailleurs le test de Tukey (Valeur critique du d de Tukey : 4,411) indique 6 modalités de $\mathrm{T}^{\circ} \mathrm{Csol}$ soit Sep (a)> Oct (b) $>$ Nov (c ) $>$ Avr; Mar; Mai (d) $>$ Dec ( e ) $>$ Fev; Jan (f) (Fig. 5).

\section{DISCUSSION}

Le sol étudié (Tableau 4) a en moyenne une très mauvaise porosité (Baize et al., 1995). Le pH est alcalin (Baize, 2000), puis qu'il est fortement calcaire (Baize, 2000). Ce sol est non salin (Durand, 1983). Par contre il est bien pourvu en M.O et riche en azote (Soltner, 1992). Par ailleurs, le rapport $\mathrm{C} / \mathrm{N}$ étant supérieur à 12 (Tableau 4), traduit une mauvaise minéralisation (Baize, 2000). Quant à La texture du sol, elle est en moyenne sabloneux-limoneuse.

Dans notre étude nous avons collecté 1303 individus et identifié 12 groupes de microarthropodes (Tableau 5), Parisi et al., 2005 , ont trouvé 18 et 13 groupes de microarthropodes respectivement dans une étude à Parma (nord de l'Italie) et une autre à Crémone (nord de l'Italie). Ainsi les microarthropodes collectés sont dominés par la présence d'acariens (49\%) et des collemboles (34\%) (Tableau 5). Ces résultats sont corroborés avec ceux du Madej et al., 2011 qui ont trouvée dans une étude sur l'évaluation de l'indice de la qualité biologique du sol que la présence des microarthropodes est dominée par les acariens suivi par les collemboles. D'après Spencer (1951) in 


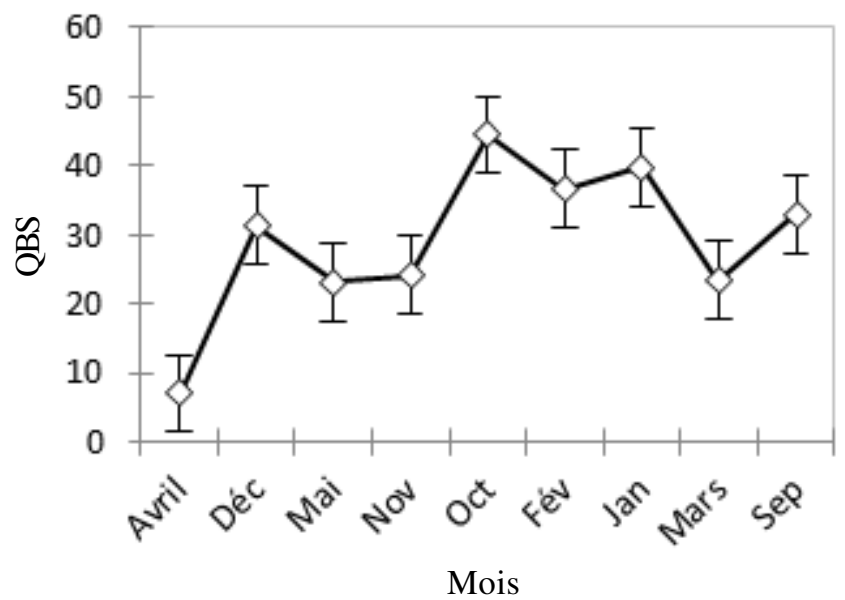

Figure 3. Variation du QBS dans les mois étudiés.

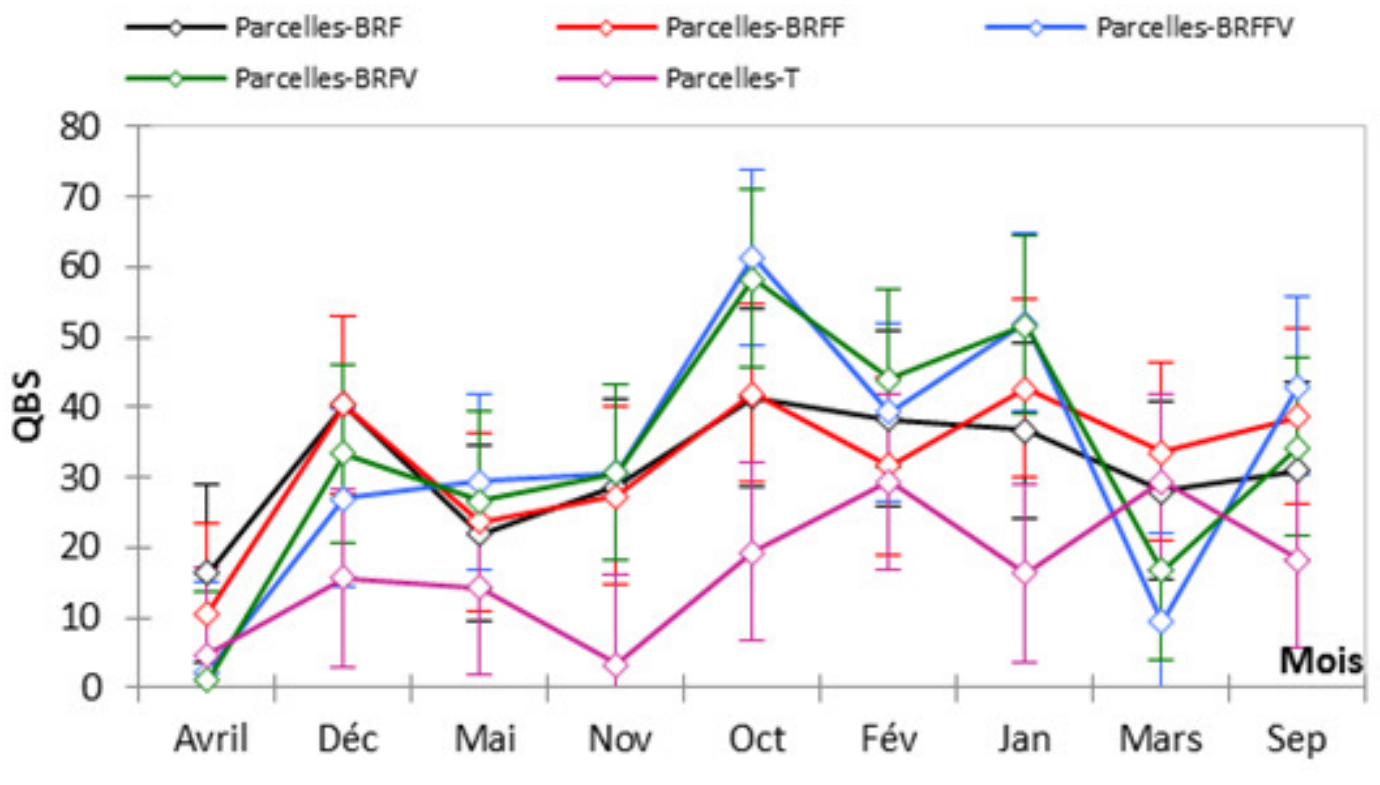

Figure 4. Variation du QBS dans les mois et les parcelles étudiés.

TABLEAU 9. Anova pour la variable température du sol

\begin{tabular}{lccccc}
\hline Source & DDL & Somme des carrés & Moyenne des carrés & $F$ & Pr $>F$ \\
\hline Mois & 8 & 13660,210 & 1707,526 & 151,144 & $<0,0001$ \\
\hline
\end{tabular}




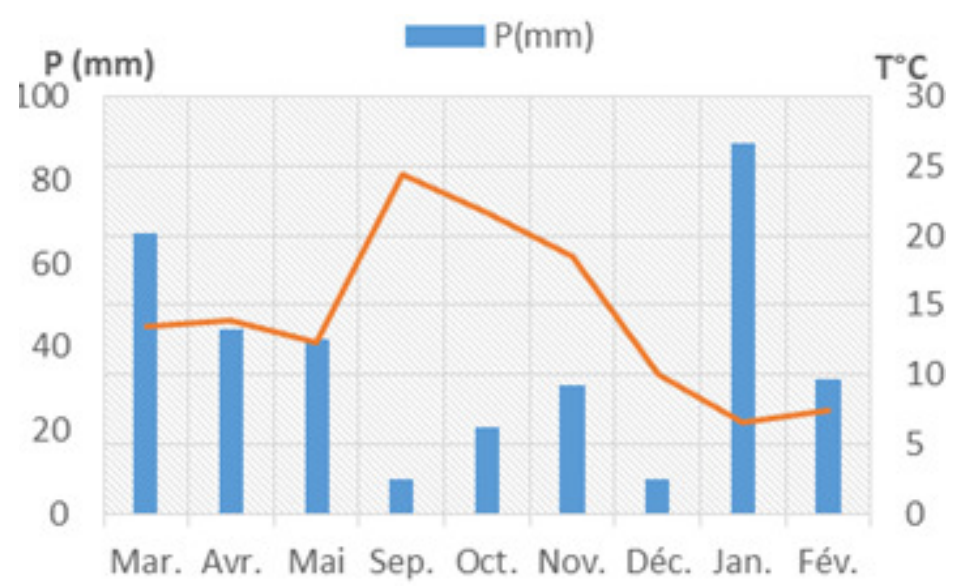

Figure 5. Variation de la $\mathrm{T}^{\circ} \mathrm{C}$ sol et des Précipitations dans les mois prospectés.

Bachelier (1963) après les acariens, les collemboles sont les arthropodes les plus nombreux du sol.

Concernant la détermination du QBS (Tableau 5) nous avons obtenu $[16,7 \pm 0,8]$; $[31,4 \pm 1,0] ;[32,3 \pm 0,8] ;[32,9 \pm 1,4]$ et $[32,7 \pm 1,4]$ respectivement dans $T, B R F, B R F F$, BRFV et BRFFV. Parisi et al., 2005 ont trouvé des valeurs du QBS de 125, 98 et de 96 dans 3 différentes études en Italie. En outre, Santorufo et al., 2012 ont trouvé une moyenne de QBS de 106 dans des sols de jardins et de parcs à Naples (Sud de l'Italie). Dans notre expérimentation, l'apport du BRF et de fumier a influencé positivement les populations des microarthropodes et donc sur le niveau du QBS calculé dans les différents types de placettes étudiées (Tableau 6). En effet, le QBS est multiplié par 2 par rapport au témoin dans les différentes placettes étudiées (Fig. 8). L'apport du BRF a favorisé l'implantation d'une microflore plus diversifiée dont la faune du sol aurait profité, et plus particulièrement les acariens et les collemboles (Larochelle, 1994). Nos résultats sont corroborés par ceux de Parisi et al., 2005, qui ont trouvés que l'apport des eaux usées sur des sols de Crémone (Italie) améliorent significativement la biologie du sol. en effet le QBS passe d'une moyenne de 82 (sols non traités) à 113 (sols traités aux eaux usées) (Parisi et al., 2005). Par ailleurs,
Larochelle, 1994, a noté que l'apport du BRF influence les microarthropodes du sol, ils étaient 5 fois plus abondantes que dans le témoin.

Concernant, l'effet de la $\mathrm{T}^{\circ} \mathrm{C}$ sol sur le QBS nous avons prélevé une température de $[13,4 \pm 0,1] ; \quad[14,0 \pm 0,1] ; \quad[12,4 \pm 0,3] ;$ $[24,4 \pm 0,1] ; \quad[21,7 \pm 0,1] ; \quad[18,5 \pm 0,1] ;$ $[10,0 \pm 0,1] ;[6,6 \pm 0,1]$ et $[7,5 \pm 0,1]{ }^{\circ} \mathrm{C}$ respectivement pour Mars, Avril, Mai, Septembre, Octobre, Novembre, Décembre, Janvier et Février, soit un QBS moyen de 28, pendant les 9 mois d'étude. Par contre, au nord de l'Italie (région humide) et pour les mois de Mars, Avril, Novembre et Décembre 2010 (Aspetti et al., 2010), ont obtenu une moyenne du QBS de 74,0 dans des champs cultivés. En outre (Andrés et al., 2011) ont trouvé une moyenne de 166 dans une étude à Vallmitjana (Taradell, Espagne). Ainsi le QBS, des régions nord du bassin méditerranéen (Italie (74) et Espagne (166)) sont supérieures à celui du QBS que nous avons estimé dans une région sud méditerranéenne. Ceci peut être expliqué par les faiblesses des pluviosités pendant la période d'étude. En effet, Il est à observer (Fig. 6) la sécheresse pendant la saison automnale de la période d'étude. Le manque de pluies a dû certainement jouer sur la diversité et l'abondance des microarthropodes et donc sur le niveau du QBS 
calculé (29). En effet l'humidité a un grand rôle sur la croissance des organismes. En fait, les fortes températures agissent surtout indirectement en desséchant le milieu, ce qui, pour les téguments fragiles et la respiration cutanée de la plupart des animaux du sol, est catastrophique. L'humidité est très importante pour les acariens, dont de nombreuses espèces supportent très bien les inondations temporaires du sol. Les collemboles supportent d'autant plus mal les fortes températures qu'ils sont soumis à un climat sec qui les déshydratent (Bachelier, 1963). L'humidité du sol demeure le facteur essentiel ayant une influence directe sur la pullulation et le comportement de la faune du sol (Ferrahi et Djema, 2004).

\section{CONCLUSION}

Cette étude s'intéresse à l'apport d'entrants énergétique (BRF et fumier) sur un sol inculte et à leurs influencent sur l'indice biologique des sols (QBS). La station d'étude est située dans une région semi-aride méditerranéenne (Algérie). Par ailleurs, le QBS de 5 placettes ( $\mathrm{T}, \mathrm{BRF}, \mathrm{BRF}, \mathrm{BRFV}$ et BRFFV) a été déterminé. L'expérimentation a duré 9 mois (Mars, Avril, Mai, Septembre, Octobre, Novembre, Décembre 2016 et Janvier et Févier 2017. Les résultats montrent que le sol étudié a en moyenne une très mauvaise porosité, un $\mathrm{pH}$ alcalin il est fortement calcaire et le rapport $\mathrm{C} / \mathrm{N}$ traduit une mauvaise minéralisation. En outre, sur un effectif de 1303 individus de microarthropodes récoltés, nous avons identifié 12 groupes de microarthropodes dont les Acariens, Collemboles, Coléoptères, Hyménoptères, Araignée, Diptere Diptères, Hemiptera, Symphyles, Psocoptera, Blattaria, Chilopode et Diplopode. Dont les acariens et les collemboles représentent $84 \%$ de l'effectif. Concernant le QBS, nous avons obtenus pour les parcelles étudiées la moyenne de $[16,7 \pm 0,8]$ ; $[31,4 \pm 1,0] ;[32,3 \pm 0,8] ;[32,9 \pm 1,4]$ et $[32,7 \pm 1,4]$ respectivement dans $\mathrm{T}, \mathrm{BRF}, \mathrm{BRFF}$, $B R F V$ et BRFFV, la différence est très significative et cela indique clairement que l'apport du BRF et de fumier influencent positivement les populations des microarthropodes et donc sur le niveau du QBS. En effet, le QBS est multiplié par 2 par rapport au témoin T. Concernant les QBS calculé pour les mois étudiés la moyenne estimée est de $[23,4 \pm 1,6] ;[7 \pm 1,6]$; $[23,2 \pm 0,8] ; \quad[33,0 \pm 1,7] ; \quad[44,4 \pm 2,4]$; $[24,1 \pm 1,6] ; \quad[31,3 \pm 1,7] ;[39,8 \pm 2,5]$ et $[36,5 \pm 1,1]$ respectivement pour le mois de Mars; Avril; Mai; Septembre, Octobre, Novembre, Décembre, Janvier et Février. La différence est très significative (Fobs $=15,573$ ; ddl $=8 \mathrm{p}<0,000)$. Par ailleurs, il y a un effet Parcelles et Mois (Fobs $=1,846$; ddl $=32$ $\mathrm{P}<0,013)$, cela signifie que le QBS est influencé par la qualité de l'apport mais aussi par les facteurs climatique tels que les précipitations et les températures. En effet, les températures du sol mensuelles $\left(\mathrm{T}^{\circ} \mathrm{C}\right.$ sol) varient très significativement d'un mois à un autre. Le QBS moyen estimé pour le sol inculte étudié est de 29. En revanche, le QBS, des régions nord du bassin méditerranéen Italie (74) et Espagne (166) sont supérieurs à celui du QBS que nous avons estimé (29). La sécheresse de la période d'étude a impacté la diversité et l'abondance des microarthropodes et donc sur le faible QBS estimé dans notre station d'étude située en zone semi-aride méditerranéenne.

\section{RÉFÉRENCES BIBLIOGRAPHIQUES}

Allison, F. E. 1973. Soil organic matter and its role in crop production. Elsevier Scientific Publishing Co, Amsterdam. 637pp.

Andrés, P., Mateos, E., Tarrasón, D., Cabrera, C. and Figuerola, B. 2011. Effects of digested composted and thermally dried sewage sludge on soil microbiota and mesofauna. Applied Soil Ecology 48: 236242.

Aspetti, G., Boccelli, R., Ampollini, D., Del Re Attilio, A.M. and Capri, E. 2010. Assessment of soil-quality index based on microarthropods in corn cultivation in 
Northern Italy. Ecological Indicators 10: 129-135.

Bachelier, G. 1963. Lavie animale dans les sols. 1éd, O.R.S.T.O.M. Paris. 279pp.

Bachelier, G. 1978. La faune du sol, son action, Initiation, Documentation technique, $\mathrm{N}^{\circ} 38$, O.R.S.T.O.M. 391pp.

Baize, D. 2000.Guide des analyses en pédologie.2éd, INRA, Paris. 257pp.

Baize, D. et Jabiole, B. 1995. Guide pour la description des sols. Ed, INRA, Paris. 375pp.

Bouche, M.B.1974. Les lombricien : Bul de Liaison. Centre Universitaire. Dijon: 27:1318.

Buckerfield, J.C., Lee, K.E., Davoren, C.W. and Hannay, J.N. 1997. Earthworms as indicators of sustainable production in dryland cropping in Southern Australia. Soil Biology and Biochemistry 29 : 547-554.

Caron, C., Lemieux, G., 1999. Le bois raméal pour la régénération des sols agricoles et forestiers. Echo MO19: 2.

Davis, B.N.K. 1961. Soil fauna in relation to soil formation and fertility. Nature 189 : 367-368.

Durand, J.H. 1983. Les sols Irrigables, Etude pédologique. Presses Université de France, Agence de Coopération Culturelle et Technique. 338pp.

Edmonds, R.L. 1987. Decomposition rates and nutrient dynamics in small-diameter woody litter in four forest ecosystems in Washington, U.S.A. Canadian Journal of Forest Research 17: 499- 509.

Edwards, C.A. and Bohlen, P.J. 1996. Biology and Ecology of Earthworms, Champon and Hall, London, UK.

Edwards, C.A. and Lofty, J.R. 1982. Nitrogenous fertilizers and earthworm populations in agricultural soils. Soil Biology and Biochemistry 14: 515-521.

Eijsackers, H. 1983. Soil fauna and soil microflora as possible indicators of soil pollution. Environmental Monitoring and Assessment 3: 307-316.
Ekschmitt, K., Bakonyi, G., Bongers, M., Bongers, T., Boström, S., Dogan, H., Harrison, A., Nagy, P., O’Donell, A.G., Papatheodorou, E.M., Sohlenius, B. and Stamou, G.P. 2001. Nematode community structure as indicator of soil functioning in European grassland soils. European Journal of Soil Biology 37: 263-268.

Hedlund, K. and Öhrn, M.S. 2000. Tritrophic interactions in a soil community enhance decomposition rates. Oikos 88: 585-591.

Hendrickson, O. 1987. Winter branch nutrients in northern conifers and hardwoods. Forest Science 33: 1068-1074.

Hiraoka, H. Misra, R.V et Roy R.N. 2005. Méthodes de compostage au niveau de l'exploitation agricole, FAO, Rome, Italy. $51 \mathrm{pp}$.

Larochelle, L. 1994. L'impact du bois raméal fragmenté sur la dynamique de la mésofaune du sol. M.Sc. Thèse. Université Laval, Canada. 78pp.

Lee, K.E. 1985. Earthworms: Their ecology and relationships with soils and land use. Academic Press, Sydney. 411pp.

Lemieux, G. 1986. Le bois raméal et les mécanismes de fertilité du sol, Publié par le Ministère de l'Énergie et des Ressources et la Faculté de Foresterie de l'Université Laval, Québec. 20pp.

Madej, G., Barczyk, G. and Gdawiec, M. 2011. Evaluation of soil biological quality index (QBS-ar): Its sensitivity and usefulness in the post mining chronosequence preliminary research. Polish Journal of Environmental Studies 5: 1367-1372.

Menta, C., Leoni, A., Bardini, M., Gardi, C. and Gatti, F. 2008. Nematode and microarthropod communities: comparative use of soil quality bioindicators in covered dump and natural soils. Environmental Bioindicators 3, 35e46. Nortcliff, S. 2002. Standardisation of soil quality attributes. Agriculture, Ecosystems and Environment 88: 161-168.

N'Dayegamiye, A. and Angers, D. A. 1993. Organic matter characteristics and 
waterstable aggregation of a sandy loam after 9 years of wood-residue applications. Canadian Journal of Soil Science 73: 115122.

Osler, G.H.R., Sommerkorn, M. 2007. Toward a complete soil $\mathrm{C}$ and $\mathrm{N}$ cycle: Incorporating the soil fauna. Ecology 88: 1611-1621.

Paoletti, M.G.and Hassal, M. 1999. Woodlice (Isopoda: Oniscidea): Their potential for assessing sustainability and use as bioindicators. Agriculture, Ecosystems and Environment 74: 157-165.

Paolo, A.G., Raffaella, B., Danio, A., Attilio, D.R.A.M.and Ettore, C. 2010. Assessment of soil-quality index based on microarthropods in corn cultivation in Northern Italy. Ecological Indicators 10: 129-135.

Parisi, V. 1974. Soil biology and ecology, techniques of researches. Boringhieri, Torino (in Italian).

Parisi, V. 2001. The biological soil quality, a method based on microarthropods. Acta Naturalia de L'Ateneo Parmense 37: 97106.

Parisi, V., Menta, C., Gardi, C., Jacomini, C. and Mozzanica, E. 2005. Microarthropod communities as a tool to assess soil quality and biodiversity: A new approach in Italy. Agriculture, Ecosystems and Environment 105: 323-333.

Santorufo, L., Van Gestel, C., Rocco, A. and Maisto,G. 2012. Soil invertebrates as bioindicators of urban soil quality. Environmental Pollution 161: 57-63.

Soltner D. 1992. Phytotechnie générale : les bases de la production végétale. Tome 1 : le Sol et son amélioration. Tome 2 : Climat, Météorologie, Pédologie, Bioclimatologie. Série Agronomie.

Stork, N.E. and Eggleton, P. 1992. Invertebrates as determinants and indicators of soil quality. American Journal of Alternative Agriculture 7: 38-47.

Tremblay, J. et Beauchamp, C.J. 1998. Fractionnement de la fertilisation azotée d'appoint à la suite de l'incorporation au sol de bois raméaux fragmentés: modifications de certaines propriétés biologiques et chimiques d'un sol cultivé en pomme de terre. Canadian Journal of Soil Science 78: 275 - 282.

Tullgren, A. 1918. Ein sehr einfacher Auslesgeapparat fur terricole Tierformen. Z. Angew. Ent $4: 149-150$.

Wolters, V. 2000. Invertebrate control of soil organic matter stability. Biology and Fertility of Soils 31: 1-19.

Yan, S., Singh, A.N., Fu, S., Liao, C., Wang, S., Li,Y., Cui, Y. and Huf, L. 2012. A soil fauna index for assessing soil quality. Soil Biology and Biochemistry 47: 158-165.

Yeates, G.W. 2003. Nematodes as soil indicators: Functional and biodiversity aspects. Biology and Fertility of Soils 37 : 199-210. 